\title{
Spectral properties of bipolar surfaces to Otsuki tori
}

\author{
Mikhail A. Karpukhin
}

\begin{abstract}
The $i$-th eigenvalue $\lambda_{i}$ of the Laplace-Beltrami operator on a surface can be considered as a functional on the space of all Riemannian metrics of unit volume on this surface. Surprisingly only few examples of extremal metrics for these functionals are known. In the present paper a new countable family of extremal metrics on the torus is provided.
\end{abstract}

Mathematics Subject Classification (2010). Primary 58E11; Secondary 58J50.

Keywords. Otsuki tori, extremal metric, bipolar surface.

\section{Introduction}

Let $M$ be a closed surface and $g$ be a Riemannian metric on $M$. Let us consider the associated Laplace-Beltrami operator $\Delta$ acting on the space of smooth functions on $M$,

$$
\Delta f=-\frac{1}{\sqrt{|g|}} \frac{\partial}{\partial x^{i}}\left(\sqrt{|g|} g^{i j} \frac{\partial f}{\partial x^{j}}\right) .
$$

It is well-known that the spectrum of $\Delta$ is non-negative and consists only of eigenvalues, each eigenvalue has finite multiplicity and the eigenfunctions are smooth. Let us denote the eigenvalues of $\Delta$ by

$$
0=\lambda_{0}(M, g)<\lambda_{1}(M, g) \leqslant \lambda_{2}(M, g) \leqslant \lambda_{3}(M, g) \leqslant \cdots,
$$

where eigenvalues are written with multiplicities.

The eigenvalues possess the property

$$
\lambda_{i}(M, \operatorname{tg})=\frac{\lambda_{i}(M, g)}{t}, \quad t>0 .
$$

Therefore, given a fixed surface $M$ one has $\sup \lambda_{i}(M, g)=+\infty$, where supremum is taken over the space of all Riemannian metrics on $M$. But if we consider supremum over the space of all Riemannian metrics on $M$ of unit area then the question about 
the value of $\sup \lambda_{i}(M, g)$ becomes more interesting. The alternative approach is to investigate the functionals

$$
\Lambda_{i}(M, g)=\lambda_{i}(M, g) \operatorname{Area}(M, g)
$$

It is easy to see that $\Lambda_{i}(M, g)=\Lambda_{i}(M, \operatorname{tg})$ for any $t>0$. Thus the latter question is equivalent to the question about the value of $\sup \Lambda_{i}(M, g)$, where supremum is taken over the space of all Riemannian metrics on $M$.

It is known that functionals $\Lambda_{i}(M, g)$ are bounded from above. Yang and Yau proved in the paper [22] that for an orientable surface $M$ of genus $\gamma$ the following inequality holds,

$$
\Lambda_{1}(M, g) \leqslant 8 \pi(\gamma+1) .
$$

Moreover, Korevaar proved in the paper [15] that there exists a constant $C$ such that for any $i>0$ and any compact surface $M$ of genus $\gamma$ the following inequality holds:

$$
\Lambda_{i}(M, g) \leqslant C(\gamma+1) i
$$

However, Colbois and Dodziuk proved in the paper [5] that for any manifold $M$ of dimension $\operatorname{dim} M \geqslant 3$ the functional $\lambda_{i}(M, g)$ is not bounded on the space of Riemannian metrics $g$ on $M$ of unit volume.

The functional $\Lambda_{i}(M, g)$ depends continuously on the metric $g$, but this functional is not differentiable. However, it is known that for an analytic family of metrics $g_{t}$ there exist the left and right derivatives with respect to $t$; see the papers [1], [2], and [8]. This is a motivation for the following definition; see the papers [9] and [18].

Definition 1. A Riemannian metric $g$ on a closed surface $M$ is called an extremal metric for the functional $\Lambda_{i}(M, g)$ if for any analytic deformation $g_{t}$ such that $g_{0}=g$ the following inequality holds:

$$
\left.\frac{d}{d t} \Lambda_{i}\left(M, g_{t}\right)\right|_{t=0+} \leqslant 0 \leqslant\left.\frac{d}{d t} \Lambda_{i}\left(M, g_{t}\right)\right|_{t=0-} .
$$

The detailed list of surfaces $M$ and values of index $i$ such that maximal or at least extremal metrics are known is quite short and can be found in the introduction to the paper [20].

It turns out that extremal metrics are closely related to minimal submanifolds of the spheres. Let $M \leftrightarrow \mathbb{S}^{n}$ be a minimally immersed submanifold of the unit sphere $\mathbb{S}^{n} \subset \mathbb{R}^{n+1}$. We denote by $\Delta$ the Laplace-Beltrami operator on $M$ associated with the induced metric $g$ on $M$. Let us introduce the eigenvalues counting function

$$
N(\lambda)=\#\left\{i \mid \lambda_{i}(M, g)<\lambda\right\}
$$

This function is often called the Weyl's function. The following theorem provides a general approach to finding smooth extremal metrics. 
Theorem 1 (El Soufi and Ilias [8]). Let $M \leftrightarrow \mathbb{S}^{n}$ be a minimally immersed submanifold of the unit sphere $\mathbb{S}^{n} \subset \mathbb{R}^{n+1}$. Then the metric induced on $M$ by the immersion is extremal for the functional $\Lambda_{N(2)}(M, g)$.

We also need to recall another result concerning minimal submanifolds of the sphere. This theorem can be found e.g. in the book [14].

Theorem 2. Let $M \leftrightarrow \mathbb{S}^{n}$ be a minimally immersed submanifold of the unit sphere $\mathbb{S}^{n} \subset \mathbb{R}^{n+1}$. Then the restrictions $\left.x^{1}\right|_{M}, \ldots,\left.x^{n+1}\right|_{M}$ on $M$ of the standard coordinate functions of $\mathbb{R}^{n+1}$ are eigenfunctions of the Laplace-Beltrami operator on $M$ with eigenvalue $\operatorname{dim} M$.

Thus, it is possible to take an immersed minimal surface $M$ in the sphere, then compute $N(2)$ and deduce that the metric induced on $M$ by the immersion is extremal for $\Lambda_{N(2)}(M, g)$. This approach was successfully realized for the first time by Penskoi in the papers [20] and [21] for Otsuki tori and Lawson tau-surfaces. Although, we should mention that Lapointe in the paper [16] used some of these ideas in investigation of bipolar surfaces to Lawson tau-surfaces. The work of Lapointe was inspired by the paper [12] where Jakobson, Nadirashvili and Polterovich proved that the metric on the Lawson bipolar surface $\tilde{\tau}_{3,1}$ is extremal for the functional $\Lambda_{1}(\mathbb{K} l, g)$. Later, El Soufi, Giacomini and Jazar proved in the paper [6] that this metric is the unique extremal metric.

In fact, all metrics extremal for the first positive eigenvalue are determined for surfaces of genus 0 and 1 . The case of a Klein bottle was mentioned above. In case of a sphere and a projective plane the only metrics extremal for $\Lambda_{1}$ are the standard metrics of constant curvature. For a torus there are two extremal metrics: the metric on the flat torus with a square lattice (Clifford torus) and the metric on the flat torus with an equilateral lattice. These results were obtained in the paper [9] by El Soufi and Ilias based on their paper [7].

In the present paper the extremality of the bipolar surfaces to Otsuki tori is investigated. The definition of Otsuki tori and bipolar surfaces are given in Sections 2.2 and 2.3 respectively. At this point it is sufficient to know that for every rational number $p / q$ such that

$$
(p, q)=1 \text { and } \frac{1}{2}<\frac{p}{q}<\frac{\sqrt{2}}{2}
$$

there exists a minimal immersed surface in $\mathbb{S}^{4}$ denoted by $\widetilde{O}_{\frac{p}{q}}$. The main result of this paper is the following theorem.

Theorem 3. The bipolar surface $\widetilde{O}_{\frac{p}{q}}$ to an Otsuki torus is a torus. If $q$ is odd then the metric on $\widetilde{O}_{\frac{p}{q}}$ induced by the immersion is extremal for $\Lambda_{2 q+4 p-2}\left(\mathbb{\nabla}^{2}, g\right)$. If $q$ is even then the metric induced by the immersion on $\widetilde{O}_{\frac{p}{q}}$ is extremal for $\Lambda_{q+2 p-2}\left(\mathbb{T}^{2}, g\right)$. 
The paper is organized in the following way. The Otsuki tori and their bipolar surfaces are defined in Sections 2.2 and 2.3. A convenient parametrization of bipolar surfaces is given in Section 2.4. Section 3 contains the proof of the main theorem.

Acknowledgments. The author thanks A. V. Penskoi for statement of this problem, fruitful discussions and invaluable help in the preparation of the manuscript.

\section{Bipolar surfaces to Otsuki tori.}

2.1. Reduction theorem for minimal submanifolds. Let $M$ be a Riemannian manifold equipped with a metric $g^{\prime}$ and $I(M)$ be its full isometry group. Let $G \subset I(M)$ be a compact isometry group. Let us denote by $\pi$ the natural projection

$$
\pi: M \longrightarrow M / G
$$

Denote by $M^{*}$ the union of all orbits of principal type, then $M^{*}$ is an open dense submanifold of $M$. The subset $M^{*} / G$ of $M / G$ is equipped with a natural Riemannian metric $g$ defined by the formula

$$
g(X, Y)=g^{\prime}\left(X^{\prime}, Y^{\prime}\right),
$$

where $X, Y$ are tangent vectors at $x \in M^{*} / G$ and $X^{\prime}, Y^{\prime}$ are tangent vectors at a point $x^{\prime} \in \pi^{-1}(x) \subset M^{*}$ such that $X^{\prime}$ and $Y^{\prime}$ are orthogonal to the orbit $\pi^{-1}(x)$ and $d \pi\left(X^{\prime}\right)=X, d \pi\left(Y^{\prime}\right)=Y$.

Let

$$
f: N \leftrightarrow M
$$

be a $G$-invariant immersed submanifold, i.e. a manifold equipped with an action of $G$ by isometries such that

$$
g \cdot f(x)=f(g \cdot x), \quad x \in N .
$$

Definition 2. The cohomogeneity of a $G$-invariant immersed submanifold $N$ is the number $\operatorname{dim} N-v$, where $v$ is the dimension of the orbits of principal type.

Let us define for $x \in M^{*} / G$ a volume function $V(x)$ by the formula

$$
V(x)=\operatorname{Vol}\left(\pi^{-1}(x)\right) .
$$

Also for each integer $k \geqslant 1$ let us define a metric

$$
g_{k}=V^{\frac{2}{k}} g \text {. }
$$

Proposition 1 (Hsiang and Lawson [11]). Let $f: N \leftrightarrow M^{*}$ be a G-invariant immersed submanifold of cohomogeneity $k$, and let $M^{*} / G$ be equipped with the metric $g_{k}$. Then $f: N \leftrightarrow M^{*}$ is minimal if and only if $\bar{f}: N / G \leftrightarrow M^{*} / G$ is minimal. 
2.2. Otsuki tori. Otsuki tori were introduced by Otsuki in the paper [19]. Let us recall the concise description by Penskoi from the paper [20]. For more details see Section 1.2 of the paper [20]. Consider the action of $\mathrm{SO}(2)$ on the three-dimensional unit sphere $\mathbb{S}^{3} \subset \mathbb{R}^{4}$ given by the formula

$$
\alpha \cdot(x, y, z, t)=(\cos \alpha x+\sin \alpha y,-\sin \alpha x+\cos \alpha y, z, t),
$$

where $\alpha \in[0,2 \pi)$ is a coordinate on $\operatorname{SO}(2)$. The space of orbits $\mathbb{S}^{3} / \mathrm{SO}(2)$ is the closed half-sphere $\mathbb{S}_{+}^{2}$,

$$
q^{2}+z^{2}+t^{2}=1, \quad q \geqslant 0
$$

where a point $(q, z, t)$ corresponds to the orbit $(q \cos \alpha, q \sin \alpha, z, t) \in \mathbb{S}^{3}$. The space of principal orbits $\left(\mathbb{S}^{3}\right)^{*} / \mathrm{SO}(2)$ is the open half sphere $\mathbb{S}_{>0}^{2}=\left\{(q, z, t) \in \mathbb{S}^{2} \mid q>0\right\}$. It is natural to introduce the spherical coordinates in the space of orbits,

$$
\left\{\begin{array}{l}
t=\cos v \sin \lambda \\
z=\cos v \cos \lambda \\
q=\sin v
\end{array}\right.
$$

Since we look for minimal submanifolds of cohomogeneity 1, the Hsiang-Lawson's metric is given by the formula

$$
V^{2}\left(d \nu^{2}+\cos ^{2} v d \lambda^{2}\right)=4 \pi^{2} \sin ^{2} v\left(d \nu^{2}+\cos ^{2} v d \lambda^{2}\right) .
$$

Definition 3. An immersed minimal $S O(2)$-invariant two-dimensional torus in $\mathbb{S}^{3}$ such that its image by the projection $\pi: \mathbb{S}^{3} \rightarrow \mathbb{S}^{3} / \mathrm{SO}(2)$ is a closed geodesics in $\left(\mathbb{S}^{3}\right)^{*} / \mathrm{SO}(2)$ equipped with metric (1) is called an Otsuki torus.

The following proposition can be found in the paper [20].

Proposition 2. Except one particular case given by the equation $\psi=\frac{\pi}{4}$, Otsuki tori are in one-to-one correspondence with rational numbers $\frac{p}{q}$ such that

$$
\frac{1}{2}<\frac{p}{q}<\frac{\sqrt{2}}{2}, \quad p, q>0,(p, q)=1 \text {. }
$$

Definition 4. By $O \frac{p}{q}$ we denote the Otsuki torus corresponding to $\frac{p}{q}$. Following the paper [20] we reserve the term Otsuki tori for the tori $O \frac{p}{q}$.

In order to fix notations we give a sketch of the proof of Proposition 2. 
Proof. Let us use the standard notation for the coefficients of metric (1),

$$
E=4 \pi^{2} \sin ^{2} v, \quad G=4 \pi^{2} \sin ^{2} v \cos ^{2} v .
$$

The equation of geodesics for $\ddot{\lambda}$ reads

$$
\ddot{\lambda}+\frac{1}{G} \frac{\partial G}{\partial v} \dot{v} \dot{\lambda}=0 .
$$

Hence, $2 \pi c=G \dot{\lambda}$ is an integral of motion and

$$
\dot{\lambda}=\frac{c}{2 \pi \cos ^{2} v \sin ^{2} v} .
$$

As we know the velocity vector of a geodesic has a constant length. Suppose this length equals 1 . Then

$$
E \dot{v}^{2}+G \dot{\lambda}^{2}=1 \Longleftrightarrow \dot{v}^{2}=\frac{\sin ^{2} v \cos ^{2} v-c^{2}}{4 \pi^{2} \sin ^{4} v \cos ^{2} v} .
$$

This implies $\sin ^{2} v \cos ^{2} v-c^{2} \geqslant 0$ and $\sin ^{2} v \cos ^{2} v=c^{2}$ if and only if $\dot{v}=0$.

Since the point corresponding to $v=0$ does not belong to $\left(\Phi^{3}\right)^{*} / \mathrm{SO}(2)$, there exists a minimal value $a$ of the coordinate $v$ on a geodesic. Therefore $c= \pm \sin a \cos a$ and the geodesics are situated in the annulus $a \leqslant v \leqslant \frac{\pi}{2}-a$. We choose a natural parameter $t$ such that $v(0)=a$.

Equations (2) and (3) imply

$$
\frac{d \nu}{d \lambda}= \pm \cos v \frac{\sqrt{\sin ^{2} v \cos ^{2} v-\sin ^{2} a \cos ^{2} a}}{\sin a \cos a} .
$$

The right hand side of this equation equals 0 only at $v=a$ and $v=\frac{\pi}{2}-a$.

Let us denote by $\Omega(a)$ the distance between the value of $\lambda$ corresponding to $v=a$ and the closest to it value of $\lambda$ corresponding to $v=\frac{\pi}{2}-a$. It is clear that

$$
\Omega(a)=\sin a \cos a \int_{a}^{\frac{\pi}{2}-a} \frac{d v}{\cos v \sqrt{\sin ^{2} v \cos ^{2} v-\sin ^{2} a \cos ^{2} a}} .
$$

The geodesic is closed if and only if $\Omega(a)=\frac{p}{q} \pi$. The rest of the proof follows from the properties of the function $\Omega(a)$ :

$$
\Omega(a) \text { is continuous and monotonous on }\left(0, \frac{\pi}{4}\right] \text {, }
$$

and

$$
\lim _{a \rightarrow 0+} \Omega(a)=\frac{\pi}{2} \quad \text { and } \quad \Omega\left(\frac{\pi}{4}\right)=\frac{\pi}{\sqrt{2}}
$$

see the paper [19]. 
The Otsuki tori $O_{\frac{p}{q}}$ are minimally immersed into $\mathbb{S}^{3}$ by the map

$$
I_{a}:[0,2 \pi) \times[0, \tilde{t}) \longrightarrow \mathbb{R}^{4}
$$

given by

$$
I_{a}(\alpha, t)=(\cos \alpha \sin v(t), \sin \alpha \sin v(t), \cos v(t) \cos \lambda(t), \cos v(t) \sin \lambda(t)),
$$

where $\Omega(a)=\frac{p}{q} \pi$ and $t$ is a natural parameter on the corresponding closed geodesic $\pi\left(O_{\frac{p}{q}}\right)$ such that

$$
\min _{t} v(t)=v(0)=a
$$

and $\tilde{t}$ is the length of this geodesic.

2.3. Construction of bipolar surfaces. Following the papers [13] and [17], we define the surface $\widetilde{O}_{\frac{p}{q}}$ bipolar to $O_{\frac{p}{q}}$ as an exterior product of $I$ and $I^{*}$, where $I^{*}$ is a unit vector normal to the torus $O_{\frac{p}{q}}$ and tangent to $\$^{3}$. By a straightforward computation one obtains

$$
\begin{aligned}
I_{a}^{*}=2 \pi \sin v & \left(\dot{\lambda} \cos ^{2} v \cos \alpha,\right. \\
& \dot{\lambda} \cos ^{2} v \sin \alpha, \\
& \dot{v} \sin \lambda-\dot{\lambda} \cos v \sin v \cos \lambda, \\
& -\dot{v} \cos \lambda-\dot{\lambda} \cos v \sin v \sin \lambda),
\end{aligned}
$$

where the dot denotes the derivative with respect to $t$, and

$$
\begin{aligned}
I_{a} \wedge I_{a}^{*}=2 \pi \sin v & (0, \\
& \cos \alpha(\dot{\lambda} \cos \lambda \cos v-\dot{v} \sin \lambda \sin v), \\
& \cos \alpha(\dot{\lambda} \sin \lambda \cos v+\dot{v} \cos \lambda \sin v), \\
& \sin \alpha(\dot{\lambda} \cos \lambda \cos v-\dot{v} \sin \lambda \sin v), \\
& \sin \alpha(\dot{\lambda} \sin \lambda \cos v+\dot{v} \cos \lambda \sin v), \\
& \dot{v} \cos v)
\end{aligned}
$$

The parametrized surface $I_{a} \wedge I_{a}^{*}$ is a minimal (see a proof in the paper [17]) immersed submanifold in the equator $\mathbb{S}^{4} \subset \mathbb{\$}^{5}$. But formula (4) is inconvenient. In the next section another parametrization of $\widetilde{O}_{\frac{p}{q}}$ is proposed.

2.4. Parametrization of $\widetilde{\boldsymbol{O}}_{\frac{p}{q}}$. Let us now apply the Hsiang-Lawson's reduction theorem (Proposition 1) in the case of $M=\$^{4}$ and $G=\operatorname{SO}(2)$. Let $x, y, z, u, v$ 
be the standard coordinates in $\mathbb{R}^{5}$ and $\mathbb{S}^{4}$ be the standard unit sphere in $\mathbb{R}^{5}$. Let us consider an action of $\mathrm{SO}(2)$ given by the formula

$$
\begin{aligned}
\alpha \cdot(x, y, z, u, v)=( & \cos \alpha x-\sin \alpha y, \\
& \sin \alpha x+\cos \alpha y, \\
& \cos \alpha z-\sin \alpha u, \\
& \sin \alpha z+\cos \alpha u, v),
\end{aligned}
$$

where $\alpha \in[0,2 \pi)$ is a coordinate on $\mathrm{SO}(2)$.

The principal orbits are circles of radius $\sqrt{x^{2}+y^{2}+z^{2}+u^{2}}$, the exceptional orbits are the poles $N=(0,0,0,0,1)$ and $S=(0,0,0,0,-1)$. It is easy to see, that for each principal orbit there are exactly two points on the equatorial sphere $\mathbb{S}^{3}$ of the unit sphere $\mathbb{S}^{4}$ given by the equation $y=0$. Therefore, the space of orbits $\left(\mathbb{S}^{4}\right)^{*} / \mathrm{SO}(2)$ can be identified with the quotient of this equatorial sphere $\mathbb{S}^{3}$ by the action of $\mathbb{Z}_{2}$ given by

$$
\sigma(x, 0, z, u, v)=(-x, 0,-z,-u, v)
$$

where $\sigma$ is the nontrivial element of $\mathbb{Z}_{2}$. Let us call the equatorial sphere given by the equation $y=0$ a generalized space of orbits. Let us denote by $p$ the quotient map from the generalized space of orbits to the space of orbits,

$$
p: \mathbb{S}^{3} \backslash\{N, S\} \longrightarrow\left(\mathbb{S}^{4}\right)^{*} / \mathrm{SO}(2) .
$$

Let us denote by $\tilde{\pi}$ the natural projection of $\left(\Phi^{4}\right)^{*}$ onto the space of orbits.

Let $g_{1}$ be the Hsiang-Lawson's metric on the space of orbits. The preimage $p^{-1}(s)$ of a closed geodesic $s$ in the space of orbits is either a closed geodesic $\gamma$ in $\left(\mathbb{S}^{3} \backslash\{N, S\}, p^{*} g_{1}\right)$ such that $\sigma \gamma=\gamma$, or a pair of closed geodesics $\left\{\gamma_{1}, \gamma_{2}\right\}$ in $\left(\mathbb{S}^{3} \backslash\{N, S\}, p^{*} g_{1}\right)$ such that $\sigma \gamma_{1}=\gamma_{2}$. Thus, each geodesic in the space of orbits is the image $p(\gamma)$ of some geodesic $\gamma$ in the generalized space of orbits.

It is useful to introduce the spherical coordinates in the generalized space of orbits,

$$
\left\{\begin{array}{l}
x=\cos \varphi \sin \theta, \\
z=\cos \varphi \cos \theta \cos \rho, \\
u=\cos \varphi \cos \theta \sin \rho, \\
v=\sin \varphi .
\end{array}\right.
$$

Then the pullback of the volume function to the generalized space of orbits is given by the formula

$$
V(\varphi, \theta, \rho)=2 \pi \cos \varphi
$$


These coordinates induce coordinates on $\$^{4}$ by the following formulae

$$
\left\{\begin{array}{l}
x=\cos \alpha \cos \varphi \sin \theta \\
y=\sin \alpha \cos \varphi \sin \theta \\
z=\cos \alpha \cos \varphi \cos \theta \cos \rho-\sin \alpha \cos \varphi \cos \theta \sin \rho \\
u=\sin \alpha \cos \varphi \cos \theta \cos \rho+\cos \alpha \cos \varphi \cos \theta \sin \rho \\
v=\sin \varphi
\end{array}\right.
$$

where $\alpha \in[0, \pi)$. The metric on $\mathbb{S}^{4}$ is given by the formula,

$$
\cos ^{2} \varphi d \alpha^{2}+d \varphi^{2}+\cos ^{2} \varphi d \theta^{2}+\cos ^{2} \varphi \cos ^{2} \theta\left(d \alpha d \rho+d \rho^{2}\right),
$$

and the induced metric on the generalized space of orbits is given by the formula,

$$
g=d \varphi^{2}+\cos ^{2} \varphi d \theta^{2}+\cos ^{2} \varphi \cos ^{2} \theta \sin ^{2} \theta d \rho^{2} .
$$

Minimal SO(2)-invariant submanifolds of cohomogeneity 1 of the sphere $\mathbb{S}^{4}$ correspond to closed geodesics in the space of orbits $\left(\mathbb{S}^{4}\right)^{*} / \mathrm{SO}(2)$. According to the discussion at the beginning of this section, in order to find these submanifolds it is sufficient to find closed geodesics in $\mathbb{S}^{3} \backslash\{N, S\}$ equipped with the metric

$$
g_{1}=V^{2} g=4 \pi^{2} \cos ^{2} \varphi\left(d \varphi^{2}+\cos ^{2} \varphi d \theta^{2}+\cos ^{2} \varphi \cos ^{2} \theta \sin ^{2} \theta d \rho^{2}\right) .
$$

Indeed, for any closed geodesic $s$ in the space of orbits there exists a closed geodesic $\gamma$ in the generalized space of orbits such that $p(\gamma)=s$. Therefore, the minimal submanifold $\tilde{\pi}^{-1}(s)$ coincides with the submanifold $\tilde{\pi}^{-1}(p(\gamma))$. Moreover, the image by $p$ of a geodesic in the generalized space of orbits is a geodesic in the space of orbits. Hence, the set of submanifolds $\tilde{\pi}^{-1}(p(\gamma))$ is exactly the set of minimal $\mathrm{SO}(2)$-invariant submanifolds of cohomogeneity 1.

Since the coefficients of the metric $g_{1}$ do not depend on $\rho$, the 2-dimensional sphere defined by $\rho=0$ is the totally geodesic 2 -sphere equipped with the metric

$$
\tilde{g}_{1}=4 \pi^{2} \cos ^{2} \varphi\left(d \varphi^{2}+\cos ^{2} \varphi d \theta^{2}\right) .
$$

Let us now look for minimal submanifolds of the special type. Consider the sphere $\mathbb{S}^{2} \subset \mathbb{S}^{4}$ defined by $y=0, \rho=0$. Then for a closed geodesic $\gamma(t)=(\varphi(t), \theta(t))$ in the space $\left(\mathbb{S}^{2} \backslash\{N, S\}, \tilde{g}_{1}\right)$ one has the corresponding immersed minimal submanifold $\tilde{\pi}^{-1}(p(\gamma))$ in $\mathbb{S}^{4}$. The immersion $J$ is given by the formula

$$
\left\{\begin{array}{l}
x=\cos \alpha \cos \varphi(t) \sin \theta(t) \\
y=\sin \alpha \cos \varphi(t) \sin \theta(t) \\
z=\cos \alpha \cos \varphi(t) \cos \theta(t) \\
u=\sin \alpha \cos \varphi(t) \cos \theta(t) \\
v=\sin \varphi(t)
\end{array}\right.
$$

where $\alpha \in[0,2 \pi)$. 
Proposition 3. The set of bipolar surfaces $\widetilde{O}_{\frac{p}{q}}$ coincides with the set of minimal surfaces $\tilde{\pi}^{-1}(p(\gamma)) \subset \mathbb{S}^{4}$, where $\gamma$ is a closed geodesic in the space $\left(\mathbb{S}^{2} \backslash\{N, S\}, \tilde{g}_{1}\right)$.

Proof. In the same way as in the proof of Proposition 2, one obtains

$$
\dot{\theta}=\frac{\cos ^{2} b}{2 \pi \cos ^{4} \varphi}
$$

and

$$
\dot{\varphi}= \pm \frac{\sqrt{\cos ^{4} \varphi-\cos ^{4} b}}{2 \pi \cos ^{3} \varphi} .
$$

By $J_{b}(\alpha, t)$ denote the immersion of the minimal submanifold corresponding to a geodesic such that the minimal value of $\varphi(t)$ equals to $b$, where $t$ is a natural parameter on the geodesic $\tilde{\pi}\left(J_{b}\right)$. Let us show that for any point $\gamma(t)$ on the geodesic $\pi\left(I_{a}\right)$ there exists a neighborhood $U$ of a point $t \in \mathbb{R} /(l \mathbb{Z})$, where $l$ is the length of the geodesic $\pi\left(I_{a}\right)$, and a function $\tau(t)$ defined on $U$, such that

$$
I_{a} \wedge I_{a}^{*}(\alpha, \tau(t))=J_{b(a)}(\alpha, t),
$$

where $\cos ^{4} b(a)=4 \sin ^{2} a \cos ^{2} a$. Comparing equations (4) and (7) one obtains

$$
\sin \varphi(t)=2 \pi \dot{v}(\tau(t)) \cos v(\tau(t)) \sin v(\tau(t)) .
$$

Let us consider the case $\dot{v}>0$ and $\dot{\varphi}>0$. On the one hand, using formula (3), one has

$$
\sin \varphi(t)=\frac{\sqrt{\cos ^{2} v(\tau(t)) \sin ^{2} v(\tau(t))-c^{2}}}{\sin v},
$$

where $c=\sin a \cos a$. Applying $\frac{d}{d t}$ to equation (11) and using formula (3) one obtains

$$
\dot{\varphi}(t) \cos \varphi(t)=\dot{\tau}(t) \frac{c^{2}-\sin ^{4} v(\tau(t))}{2 \pi \sin ^{4} v(\tau(t)) \cos v(\tau(t))} .
$$

On the other hand, combining equations (9) and (10) one has the following formula,

$$
\dot{\varphi}(t) \cos \varphi(t)=\frac{c^{2}-\sin ^{4} v(\tau(t))}{2 \pi\left(\sin ^{4} v(\tau(t))+c^{2}\right)} .
$$

Therefore, one obtains a differential equation for $\tau(t)$,

$$
\dot{\tau}=\frac{\sin ^{4} v(\tau) \cos v(\tau)}{\sin ^{4} v(\tau)+c^{2}} .
$$

Let $\tau(t)$ be a solution of this equation. Comparing equations (4) and (7) one has the formulae 


$$
\cos \varphi(t) \sin \theta(t)=2 \pi \sin \psi(\tau(t))(\dot{\lambda} \cos \lambda \cos \psi-\dot{v} \sin \lambda \sin v)(\tau(t)),
$$
and

$$
\cos \varphi(t) \cos \theta(t)=2 \pi \sin \psi(\tau(t))(\dot{\lambda} \sin \lambda \cos \psi+\dot{v} \cos \lambda \cos v)(\tau(t)) .
$$

One should prove that for a function $\theta(t)$ defined by implicit formulae (13) differential equation (8) holds. This can be shown by a straightforward calculation (we omit it in order to shorten the paper). This completes the proof.

2.5. Properties of the new parametrization. Let us denote by $t_{0}$ the length of the geodesic $\tilde{\pi}\left(\widetilde{O}_{\frac{p}{q}}\right)$ with respect to the metric $\tilde{g}_{1}$. As coordinates on the torus $\widetilde{O}_{\frac{p}{q}}$ we take the parameter $\alpha \in[0,2 \pi)$ on $\mathrm{SO}(2)$ and a natural parameter $t \in\left[0, t_{0}\right)$ on the geodesic $\tilde{\pi}\left(\widetilde{O}_{\frac{p}{q}}\right)=(\varphi(t), \theta(t))$ such that $\min _{t} \varphi(t)=\varphi(0)=b$.

Proposition 4. The function $\sin \varphi(t)$ has exactly $2 q$ zeroes on $\left[0, t_{0}\right)$, the functions $\cos \theta(t)$ and $\sin \theta(t)$ both have exactly $2 p$ zeroes on $\left[0, t_{0}\right)$. If $q$ is even then the immersion $J_{b}$ is invariant under the transformation

$$
(\alpha, t) \longmapsto\left(\alpha+\pi, t+\frac{t_{0}}{2}\right)
$$

The immersion $J$ is not invariant under any other transformations.

Proof. Let us remark that the immersions $I_{a}$ and $J_{b}$ are well-defined even if the corresponding geodesics are not closed. We proved in Proposition 3 that the bipolar surface to $I_{a}$ corresponds to the geodesic $\tilde{\pi}\left(J_{b}\right)$, where

$$
\cos ^{4} b=4 \sin ^{2} a \cos ^{2} a .
$$

Hence, $\pi\left(I_{a}\right)$ is closed if and only if $\tilde{\pi}\left(J_{b}\right)$ is closed.

According to formula (4), the geodesic $\tilde{\pi}\left(J_{b}\right)$ admits another parametrization in terms of $\lambda(s)$ and $v(s)$, where $s$ is a natural parameter on $\pi\left(I_{a}\right)$. It is easy to see that this parametrization is one-to-one outside of self-intersection points, i.e. for the map

$$
\begin{aligned}
\beta(s)=2 \pi \sin v & (\dot{\lambda} \cos \lambda \cos v-\dot{v} \sin \lambda \sin v, \\
& \dot{\lambda} \sin \lambda \cos v+\dot{v} \cos \lambda \sin v, \\
& \dot{v} \cos v),
\end{aligned}
$$

where $s \in[0, \tilde{t})$, there is no point $\tilde{s}$ such that $\beta([0, \tilde{s}))=\beta([\tilde{s}, \tilde{t}))=\beta([0, \tilde{t}))$. Indeed, since $a \leqslant v \leqslant \frac{\pi}{2}-a$, the last coordinate is equal to zero only at zeroes of $\dot{v}(s)$, i.e. at $s_{d}=\frac{\tilde{t} d}{2 q}$, where $d=0,1, \ldots, 2 q-1$ and $\lambda\left(s_{d}\right)=\frac{p d}{q} \pi$. Hence, there exists $d=0,1, \ldots, 2 q-1$ such that $\tilde{s}=s_{d}$. Moreover, $v\left(s_{d}\right)=a$ if $d$ is even and $v\left(s_{d}\right)=\frac{\pi}{2}-a$ if $d$ is odd. The value of $2 \pi \dot{\lambda} \sin v \cos v=\sin a /(\sin v \cos v)$ is equal to $1 / \cos a$ for each point $s_{d}$. Therefore, $\cos \lambda\left(s_{d}\right)=\cos \lambda(0)=1$ and 
$\sin \lambda\left(s_{d}\right)=\sin \lambda(0)=0$. This holds for $s_{0}=0$ and possibly for $s_{q}$. In the latter case $\nu\left(s_{q}\right)=\frac{\pi}{2}-a$ and $(2 \pi \dot{v} \sin v \cos \nu)\left(s_{q}+\varepsilon\right)<0$ for sufficiently small $\varepsilon$. This contradicts the fact that $(2 \pi \dot{v} \sin v \cos v)(\varepsilon)>0$.

The previous statement implies that the function $\sin \varphi(t)$ has the same quantity of zeroes as $2 \pi \dot{v}(t) \sin v(t) \cos v(t)$, i.e. $\sin \varphi(t)$ has exactly $2 q$ zeroes.

Let us introduce a function analogous to $\Omega(a)$. The function $\Xi(b)$ equals the distance between the nearest points on the geodesic $\tilde{\pi}\left(J_{b}\right)$ with $\varphi=b$ and $\varphi=-b$,

$$
\Xi(b)=\cos ^{2} b \int_{b}^{-b} \frac{1}{\cos \varphi \sqrt{\cos ^{4} \varphi-\cos ^{4} b}} d \varphi .
$$

In Section 3.7 the following proposition is proved.

Proposition 5. The function $\Xi(b)$ is increasing and continuous on the interval $\left(-\frac{\pi}{2}, 0\right)$. The following equality holds:

$$
\lim _{b \rightarrow 0-} \Xi(b)=\frac{\sqrt{2}}{2} \pi .
$$

The geodesic $\tilde{\pi}\left(J_{b}\right)$ is closed if and only if $\Xi(b)=\frac{r}{s} \pi$, where $r, s \in \mathbb{Z}_{\geqslant 0}$. Without loss of generality one can assume that $(r, s)=1$. Since $\sin \varphi(t)$ has $2 q$ zeroes, one has $s=q$. According to formula (14), the function $b(a)$ increases as $a$ increases. So, we have two increasing continuous functions $\Omega(a)$ and $\Xi(b(a))$ such that their values at the point $a=\frac{\pi}{4}$ coincide and

$$
\Omega(a)=\frac{p}{q} \pi \Longleftrightarrow \Xi(b(a))=\frac{r}{q} \pi .
$$

We claim that such two functions coincide. Indeed, let us introduce the following sets:

and

$$
A_{\Omega}(s)=\left\{\frac{p}{s} \pi: \Omega(0)<\frac{p}{s} \pi<\Omega\left(\frac{\pi}{4}\right),(p, s)=1\right\}
$$

$$
A_{\Xi}(s)=\left\{\frac{p}{s} \pi: \Xi(b(0))<\frac{p}{s} \pi<\Xi\left(b\left(\frac{\pi}{4}\right)\right),(p, s)=1\right\} .
$$

On the one hand, condition (15) implies that $\left|A_{\Omega}(s)\right|=\left|A_{\Xi}(s)\right|$ for any $s$. On the other hand, suppose that $\Omega(0) \neq \Xi(b(0))$. Then for a sufficiently large $s$ one has $\left|A_{\Omega}(s)\right| \neq\left|A_{\Xi}(s)\right|$. This observation leads to a contradiction, hence $\Omega(0)=$ $\Xi(b(0))=\frac{1}{2} \pi$. Then $A_{\Omega}(s)=A_{\Xi}(s)$ and we denote this set simply by $A(s)$. Let us consider the inverse functions

$$
f=\Omega^{-1} \text { and } g=(\Xi \circ b)^{-1} .
$$


These functions are monotonous and continuous on the interval $\left(\frac{1}{2} \pi, \frac{\sqrt{2}}{2} \pi\right)$. Condition (15) means that for any $s$ one has $f(A(s))=g(A(s))$. By monotonicity of $f$ and $g$, we have $f(x)=g(x)$ for any $x \in A(s)$. Therefore $f$ and $g$ coincide on the dense subset $\bigcup_{s} A(s)$ of interval, hence by continuity $f(x) \equiv g(x)$ and $\Omega(a)=\Xi(b(a))$. Then, since $\Omega(a)=\Xi(b(a))$, the functions $\cos \theta(t)$ and $\sin \theta(t)$ have $2 p$ zeroes.

Since each orbit has exactly two intersection points with the generalized space of orbits, the immersion $J_{b}$ is invariant under some transformation if and only if the corresponding geodesic $\gamma=\tilde{\pi}\left(\widetilde{O}_{\frac{p}{q}}\right)$ is invariant under the action of $\mathbb{Z}_{2}$ given by formula (5). This means that $\operatorname{Im} \gamma$ contains the point $(b, \pi)$. According to the first statement of this proposition, if $\left(\varphi\left(t_{1}\right), \theta\left(t_{1}\right)\right)=\gamma\left(t_{1}\right)$ and $\varphi\left(t_{1}\right)=b$ then $\theta\left(t_{1}\right)=\frac{2 k p}{q} \pi$, where $k=0,1, \ldots, q-1$. Hence, $\theta\left(t_{1}\right)$ can be equal to $(2 l+1) \pi$ if and only if $q \equiv 0 \bmod 2$ and $k=\frac{q}{2}$. This implies that $t_{1}=\frac{t_{0}}{2}$. Since the map $(\varphi, \theta) \mapsto(\varphi, \theta+\pi)$ is an isometry of the orbit space, $J_{b}$ is invariant under the transformation $(\alpha, t) \mapsto\left(\alpha+\pi, t+\frac{t_{0}}{2}\right)$.

\section{Proof of the Theorem}

3.1. Relation to the theory of periodic Sturm-Liouville problems. In this section the eigenvalue counting problem for the Laplace-Beltrami operator on the bipolar Otsuki torus $\widetilde{O}_{\frac{p}{q}}$ is reduced to the same problem for the periodic Sturm-Liouville operator.

Proposition 6. Let $\widetilde{O}_{\frac{p}{q}}$ be a bipolar surface to an Otsuki torus $O_{\frac{p}{q}}$ parametrized by immersion $J_{b}(\alpha, t)$ as in Section 2.5. Then the corresponding Laplace-Beltrami operator is given by the formula

$$
\Delta f=-\frac{1}{\cos ^{2} \varphi(t)} \frac{\partial^{2} f}{\partial \alpha^{2}}-\frac{\partial}{\partial t}\left(4 \pi^{2} \cos ^{2} \varphi(t) \frac{\partial f}{\partial t}\right) .
$$

Proof. The metric on the sphere $\mathbb{S}^{4}$ is given by formula (6). Since $\rho=0$, the metric on $\widetilde{O}_{\frac{p}{q}}$ is given by the formula

$$
\cos ^{2} \varphi(t) d \alpha^{2}+\left(\dot{\varphi}(t)^{2}+\dot{\theta}(t)^{2} \cos ^{2} \varphi(t)\right) d t^{2} .
$$

But the length of the velocity vector of $\tilde{\pi}\left(O_{\frac{p}{q}}\right)$ is equal to 1 , therefore,

$$
4 \pi^{2} \cos ^{2} \varphi(t)\left(\dot{\varphi}(t)^{2}+\dot{\theta}(t)^{2} \cos ^{2} \varphi(t)\right)=1 .
$$

Hence the metric on $\widetilde{O}_{\frac{p}{q}}$ equals to

$$
h=\cos ^{2} \varphi(t) d \alpha^{2}+\frac{1}{4 \pi^{2} \cos ^{2} \varphi(t)} d t^{2}
$$

and formula (16) could be obtained by a direct calculation. 
Proposition 7. A number $\lambda$ is an eigenvalue of $\Delta$ if and only if there exists $l \in \mathbb{Z}_{\geqslant 0}$ and an eigenvalue $\lambda(l)$ of the following periodic Sturm-Liouville problem

$$
\left\{\begin{array}{l}
\frac{d}{d t}\left(4 \pi^{2} \cos ^{2} \varphi(t) \frac{d h(t)}{d t}\right)+\left(\lambda-\frac{l^{2}}{\cos ^{2} \varphi(t)}\right) h(t)=0, \\
h\left(t+t_{0}\right) \equiv h(t),
\end{array}\right.
$$

such that $\lambda(l)=\lambda$.

Proof. Let us remark that $\Delta$ commutes with $\frac{\partial}{\partial \alpha}$. It follows that $\Delta$ has a basis of eigenfunctions of the form $h(l, t) \cos (l \alpha)$ and $h(l, t) \sin (l \alpha)$. Substituting these eigenfunctions into the formula $\Delta f=\lambda f$ one obtains equation (18). Since

$$
f(\alpha+2 \pi, t) \equiv f\left(\alpha, t+t_{0}\right) \equiv f(\alpha, t),
$$

one has $l \in \mathbb{Z}$ and the boundary condition in formula (18).

Equation (18) is written in the classical form of the periodic Sturm-Liouville problem, and the following proposition holds, see e.g. the book [4].

Proposition 8. Consider a periodic Sturm-Liouville problem in the form

$$
-\frac{d}{d t}\left(p(t) \frac{d}{d t} h(t)\right)+q(t) h(t)=\lambda h(t)
$$

where

$$
p(t)>0, \quad p\left(t+t_{0}\right) \equiv p(t), \quad q\left(t+t_{0}\right) \equiv q(t) .
$$

Let us denote by $\lambda_{i}$ and $h_{i}(t)($ for $i=0,1,2, \ldots)$ the eigenvalues and eigenfunctions of problem (19) with the periodic boundary conditions

$$
h\left(t+t_{0}\right) \equiv h(t) .
$$

Let us also denote by $\tilde{\lambda}_{i}$ and $\tilde{h}_{i}(t)($ for $i=1,2, \ldots)$ the eigenvalues and eigenfunctions of problem (19) with antiperiodic boundary conditions

$$
h\left(t+t_{0}\right) \equiv-h(t) .
$$

Then the following inequalities hold,

$$
\lambda_{0}<\tilde{\lambda}_{1} \leqslant \tilde{\lambda}_{2}<\lambda_{1} \leqslant \lambda_{2}<\tilde{\lambda}_{3} \leqslant \tilde{\lambda}_{4}<\lambda_{3} \leqslant \lambda_{4}<\cdots .
$$

For $\lambda=\lambda_{0}$ there exists a unique (up to multiplication by a non-zero constant) eigenfunction $h_{0}(t)$. If $\lambda_{2 i+1}<\lambda_{2 i+2}$, for $i \geqslant 0$, there is a unique (up to multiplication by a non-zero constant) eigenfunction $h_{2 i+1}(t)$ with eigenvalue $\lambda_{2 i+1}$ of multiplicity 1 and there is a unique (up to multiplication by a non-zero constant) eigenfunction $h_{2 i+2}(t)$ with eigenvalue $\lambda_{2 i+2}$ of multiplicity 1 . If $\lambda_{2 i+1}=\lambda_{2 i+2}$ then there 
is two-dimensional eigenspace spanned by $h_{2 i+1}(t)$ and $h_{2 i+2}(t)$ with eigenvalue $\lambda=\lambda_{2 i+1}=\lambda_{2 i+2}$ of multiplicity 2 . The same holds in case $\tilde{\lambda}_{2 i+1}<\tilde{\lambda}_{2 i+2}$ and $\tilde{\lambda}_{2 i+1}=\tilde{\lambda}_{2 i+2}$.

The eigenfunction $h_{0}(t)$ has no zeros on $\left[0, t_{0}\right)$. The eigenfunctions $h_{2 i+1}(t)$ and $h_{2 i+2}(t)$ each have exactly $2 i+2$ zeros on $\left[0, t_{0}\right)$. The eigenfunctions $\tilde{h}_{2 i+1}(t)$ and $\tilde{h}_{2 i+2}(t)$ each have exactly $2 i+1$ zeros on $\left[0, t_{0}\right)$.

Corollary 1. Let $h_{i}(l, t)$ and $\lambda_{i}(l)$ be the $i$-th eigenfunction and the $i$-th eigenvalue of problem (18) for a fixed $l$. Then the eigenspace of the Laplace-Beltrami operator $\Delta$ with eigenvalue $\lambda$ has a basis consisting of functions of the form

$$
h_{i}(l, t) \cos (l \alpha),
$$

where $l \in \mathbb{Z} \geqslant 0$ and there exists $i$ such that $\lambda_{i}(l)=\lambda$, and

$$
h_{i}(l, t) \sin (l \alpha),
$$

where $l \in \mathbb{N}$ and there exists $i$ such that $\lambda_{i}(l)=\lambda$.

Proof. The statement follows from Propositions 7 and 8 for a fixed $l$.

3.2. Rayleigh quotient. Let us now investigate properties of eigenvalues $\lambda_{i}(l)$ as functions of $l$. One of the most efficient tools for this investigation is a Rayleigh quotient. The Rayleigh quotient for problem (19) is defined by

$$
R[v]=\frac{\int_{0}^{t_{0}} p(t) \dot{v}^{2}+q(t) v^{2} d t}{\int_{0}^{t_{0}} v^{2} d t} .
$$

The following proposition can be found e.g. in the book [10].

Proposition 9 (Variational principle). For the eigenvalue $\lambda_{0}$ of problem (19) with the boundary condition (20) one has

$$
\lambda_{0}=\inf _{v} R[v],
$$

where infimum is taken over the space of $t_{0}$-periodic functions $v \in H^{1}$.

For the first eigenvalue $\tilde{\lambda}_{1}$ of problem (19) with the boundary condition (21) one has

$$
\tilde{\lambda}_{1}=\inf _{u} R[u],
$$

where infimum is taken over the space of to-antiperiodic functions $v \in H^{1}$. 
Corollary 2. For any smooth $t_{0}$-periodic function $f$ one has the inequality

$$
\lambda_{0} \leqslant R[f]
$$

For any smooth $t_{0}$-antiperiodic function $g$ one has the inequality

$$
\tilde{\lambda}_{1} \leqslant R[g]
$$

Corollary 3. For the family of the periodic Sturm-Liouville problems in (18) one has $\lambda_{0}(l)>\lambda_{0}\left(l^{\prime}\right)$ as long as $l>l^{\prime}$.

Proposition 10. The following inequality holds,

$$
\lambda_{0}(2)>2
$$

Proof. Let us use the variational principle for problem (18) with $l=2$,

$$
\begin{aligned}
\lambda_{0}(2)=\inf _{v} \frac{\int_{0}^{t_{0}}\left(4 \pi^{2} \cos ^{2} \varphi(t) \dot{v}^{2}+\frac{4}{\cos ^{2} \varphi(t)}\right) v^{2} d t}{\int_{0}^{t_{0}} v^{2} d t} \\
\geqslant \inf _{v} \frac{\int_{0}^{t_{0}} \frac{4}{\cos ^{2} \varphi(t)} v^{2} d t}{\int_{0}^{t_{0}} v^{2} d t} \\
\geqslant 4>2 .
\end{aligned}
$$

By Theorem 2, functions (7) are eigenfunctions of the Laplace-Beltrami operator on the $\widetilde{O}_{\frac{p}{q}}$. It follows from formulae (7) that the functions $\cos \varphi(t) \sin \theta(t)$ and $\cos \varphi(t) \cos \theta(t)$ are eigenfunctions of problem (18) with $l=1$. Proposition 4 implies that both functions have exactly $2 p$ zeros. Hence, one can set $h_{2 p-1}(1, t)=$ $\cos \varphi(t) \sin \theta(t)$ and $h_{2 p}(1, t)=\cos \varphi(t) \cos \theta(t)$. In the same way $\sin \varphi(t)$ is an eigenfunction of problem (18) with $l=0$ and $\sin \varphi(t)$ has exactly $2 q$ zeros. Hence, either $h_{2 q-1}(0, t)=\sin \varphi(t)$ or $h_{2 q}(0, t)=\sin \varphi(t)$.

It turns out that the most difficult part of this paper is to prove that $h_{2 q}(0, t)=$ $\sin \varphi(t)$. 
3.3. Periods of eigenfunctions. Suppose that the coefficients $p(t), q(t)$ have a period less than $t_{0}$. We are interested in the eigenfunctions with the same period.

Proposition 11. Let $h_{i}(t)$ be the eigenfunctions of the periodic Sturm-Liouville problem in (19) and (20) with $\frac{t_{0}}{2 n}$-periodic coefficients enumerated as in Proposition 8. Then the $\frac{t_{0}}{2 n}$-antiperiodic solutions of problem (19) are $h_{2 n(2 k+1)-1}(t)$ and $h_{2 n(2 k+1)}(t)$, where $k \in \mathbb{Z}$.

Proof. Let us consider the Sturm-Liouville problem

$$
\left\{\begin{array}{l}
-\frac{d}{d t}\left(p(t) \frac{d h(t)}{d t}\right)+q(t) h(t)=\lambda h(t), \\
h(t) \equiv-h\left(t+\frac{t_{0}}{2 n}\right) .
\end{array}\right.
$$

By Proposition 8, its eigenvalues $\tilde{\lambda}_{i}$ form a sequence

$$
\tilde{\lambda}_{1} \leqslant \tilde{\lambda}_{2}<\tilde{\lambda}_{3} \leqslant \tilde{\lambda}_{4}<\cdots .
$$

Since $\frac{t_{0}}{2 n}$-antiperiodic solutions are also $t_{0}$-periodic, the corresponding eigenfunctions $\tilde{h}_{i}(t)$ are solutions of the problem in (19) and (20). The eigenfunctions $\tilde{h}_{2 i-1}(t)$ and $\tilde{h}_{2 i}(t)$ have exactly $2 i-1$ zeros on the interval $\left[0, \frac{t_{0}}{2 n}\right)$. Hence, they have $2 n(2 i+1)$ zeros on the interval $\left[0, t_{0}\right)$. There are only two solutions of equations (19) and (20) possessing this quantity of zeroes, therefore $\tilde{h}_{2 i-1}(t) \equiv h_{2 n(2 i-1)-1}(t)$ and $\tilde{h}_{2 i}(t) \equiv h_{2 n(2 i-1)}$.

The following proposition can be proved in the same way.

Proposition 12. Let $h_{i}(t)$ be the eigenfunctions of the periodic Sturm-Liouville problem in (19) and (20) with $\frac{t_{0}}{n}$-periodic coefficients enumerated as in Proposition 8. Then $\frac{t_{0}}{n}$-periodic solutions of problem (19) are $h_{0}, h_{2 n k-1}$ and $h_{2 n k}$, where $k \in \mathbb{Z}$.

3.4. Estimates for $\lambda_{2 q-1}(0)$. The main goal of this section is to prove that the inequality $\lambda_{2 q-1}(0)<2$ holds. Due to Proposition $11, \lambda_{2 q-1}(0)$ is equal to the first eigenvalue of the following problem:

$$
\left\{\begin{array}{l}
-\frac{d}{d t}\left(4 \pi^{2} \cos ^{2} \varphi(t) \frac{d h(t)}{d t}\right)=\lambda h(t), \\
h\left(t+\frac{t_{0}}{2 q}\right) \equiv-h(t) .
\end{array}\right.
$$

The application of Corollary 2 with

$$
f(t)=\sin \frac{2 q \pi t}{t_{0}}
$$


yields

$$
\begin{aligned}
& \lambda_{2 q-1}(0)<R[f] \frac{16 q^{2} \pi^{4} \int_{0}^{\frac{t_{0}}{2 q}} \cos ^{2} \varphi(t) \cos ^{2} \frac{2 q \pi t}{t_{0}} d t}{=} \\
&=\frac{t_{0}^{2} \int_{0}^{\frac{t_{0}}{2 q}} \sin ^{2} \frac{2 q \pi t}{t_{0}} d t}{t_{0}^{3}} \int_{0}^{\frac{t_{0}}{2 q}} \cos ^{2} \varphi(t)\left(1+\cos \frac{4 q \pi t}{t_{0}}\right) d t .
\end{aligned}
$$

According to Proposition 4, the integrand has a symmetry of the form

$$
t \longmapsto \frac{t_{0}}{2 q}-t
$$

and $\cos \varphi(t)$ is increasing on $\left(0, \frac{t_{0}}{4 q}\right)$. Hence, one obtains

$$
\begin{aligned}
\int_{0}^{\frac{t_{0}}{2 q}} \cos ^{2} \varphi(t) \cos \frac{4 q \pi t}{t_{0}} d t & =2 \int_{0}^{\frac{t_{0}}{4 q}} \cos ^{2} \varphi(t) \cos \frac{4 q \pi t}{t_{0}} d t \\
& <2 \cos ^{2} \varphi\left(\frac{t_{0}}{8 q}\right) \int_{0}^{\frac{t_{0}}{4 q}} \cos \frac{4 q \pi t}{t_{0}} d t \\
& =0 .
\end{aligned}
$$

Therefore, it is sufficient to prove that

$$
\frac{32 \pi^{4} q^{3}}{t_{0}^{3}} \int_{0}^{\frac{t_{0}}{2 q}} \cos ^{2} \varphi(t) d t<2 .
$$

It follows from formula (9) and Proposition 4 that $\varphi(t)$ is a smooth monotonous function on $\left(0, \frac{t_{0}}{2 q}\right)$. The obvious equality

$$
t_{0}=2 q \int_{0}^{\frac{t_{0}}{2 q}} d t
$$


holds. Thus, using the change of variables $t \rightarrow \varphi(t)$ one obtains

$$
\frac{32 \pi^{4} q^{3}}{t_{0}^{3}} \int_{0}^{\frac{t_{0}}{2 q}} \cos ^{2} \varphi(t) d t=\pi^{2} \frac{\int_{-b}^{b} \frac{\cos ^{5} \varphi}{\sqrt{\cos ^{4} \varphi-\cos ^{4} b}} d \varphi}{\left(\int_{-b}^{b} \frac{\cos ^{3} \varphi}{\sqrt{\cos ^{4} \varphi-\cos ^{4} b}} d \varphi\right)^{3}} .
$$

Hence, the question is reduced to estimating this ratio of integrals. Let us denote the numerator by $I_{1}(b)$ and the denominator by $I_{2}(b)$, where $b \in\left[0, \frac{\pi}{2}\right]$. We use notations $K, E$ and $\Pi$ for the complete elliptic integrals of first, second and third kind respectively (see e.g. the book [3]),

$$
\begin{aligned}
K(k) & =\int_{0}^{1} \frac{1}{\sqrt{1-x^{2}} \sqrt{1-k^{2} x^{2}}} d x, \\
E(k) & =\int_{0}^{1} \frac{\sqrt{1-k^{2} x^{2}}}{\sqrt{1-x^{2}}} d x, \\
\Pi(n, k) & =\int_{0}^{1} \frac{1}{\left(1-n x^{2}\right) \sqrt{1-x^{2}} \sqrt{1-k^{2} x^{2}}} d x .
\end{aligned}
$$

Proposition 13. The function $\frac{I_{1}(b)}{I_{2}^{3}(b)}$ is decreasing on $\left(0, \frac{\pi}{2}\right)$.

Proof. One has

$$
\begin{aligned}
I_{2}(b) & =\int_{-\sin b}^{\sin b} \frac{1-y^{2}}{\sqrt{\left(1-y^{2}\right)^{2}-\cos ^{4} b}} d y \\
& =\int_{-1}^{1} \frac{1-x^{2} \sin ^{2} b}{\sqrt{1-x^{2}} \sqrt{1+\cos ^{2} b-x^{2} \sin ^{2} b}} d x .
\end{aligned}
$$

Here the following changes of variables were used, $\sin \varphi=y, y=x \sin b$. In the same way

$$
I_{1}(b)=\int_{-1}^{1} \frac{\left(1-x^{2} \sin ^{2} b\right)^{2}}{\sqrt{1-x^{2}} \sqrt{1+\cos ^{2} b-x^{2} \sin ^{2} b}} d x .
$$


Let us remark that

$$
\frac{d\left(x \sqrt{1-x^{2}} \sqrt{1+\cos ^{2} b-x^{2} \sin ^{2} b}\right)}{d x}=\frac{3 x^{4} \sin ^{2} b-4 x^{2}+1+\cos ^{2} b}{\sqrt{1-x^{2}} \sqrt{1+\cos ^{2} b-x^{2} \sin ^{2} b}} .
$$

Integrating over the interval $[-1,1]$, one obtains the following equality,

$$
\frac{1}{3} \int_{-1}^{1} \frac{3 x^{4} \sin ^{2} b-4 x^{2}+1+\cos ^{2} b}{\sqrt{1-x^{2}} \sqrt{1+\cos ^{2} b-x^{2} \sin ^{2} b}} d x=0 .
$$

One can subtract this formula from the $I_{1}(b)$. Hence, the following equality holds,

$$
I_{1}(b)=\frac{2}{3} \int_{-1}^{1} \frac{\left(3-\sin ^{2} b-\sin ^{2} b \cos ^{2} b\right)-2 x^{2} \sin ^{2} b}{2 \sqrt{1-x^{2}} \sqrt{1+\cos ^{2} b-x^{2} \sin ^{2} b}} .
$$

Let us introduce the notation

$$
k^{2}=\frac{\sin ^{2} b}{1+\cos ^{2} b} .
$$

Then, it follows, that

$$
I_{1}(b)=\frac{4}{3} \sqrt{\frac{2}{1+k^{2}}}\left(E(k)-\frac{\left(1-k^{2}\right)\left(1+3 k^{2}\right)}{4\left(1+k^{2}\right)} K(k)\right)
$$

and

$$
I_{2}(b)=2 \sqrt{\frac{2}{1+k^{2}}}\left(E(k)-\frac{1-k^{2}}{2} K(k)\right) .
$$

Since $k(b)$ is an increasing function, it is sufficient to prove that $\frac{I_{1}}{I_{2}^{3}}$ is a decreasing function of $k$. Using classical formulae for the derivatives of the elliptic integrals

$$
\frac{d E(k)}{d k}=\frac{E(k)-K(k)}{k}
$$

and

$$
\frac{d K(k)}{d k}=\frac{E(k)}{k\left(1-k^{2}\right)}-\frac{K(k)}{k},
$$

one gets

$$
\frac{d I_{2}(k)}{d k}=2 \sqrt{\frac{2}{\left(1+k^{2}\right)^{3}}} \frac{1-k^{2}}{2 k}(E(k)-K(k))
$$

and

$$
\frac{d I_{1}(k)}{d k}=2 \sqrt{\frac{2}{\left(1+k^{2}\right)^{3}}} \frac{1-k^{2}}{2 k}\left(E(k)-\frac{1+3 k^{2}}{1+k^{2}} K(k)\right) .
$$


Since

$$
\left(\frac{I_{1}(k)}{I_{2}^{3}(k)}\right)^{\prime}=\frac{I_{1}^{\prime}(k) I_{2}(k)-3 I_{1}(k) I_{2}^{\prime}(k)}{I_{2}^{4}(k)},
$$

it is sufficient to prove that

$$
I_{1}^{\prime}(k) I_{2}(k)-3 I_{1}(k) I_{2}^{\prime}(k)<0 \text {. }
$$

Using two previous formulae one has

$$
I_{1}^{\prime}(k) I_{2}(k)-3 I_{1}(k) I_{2}^{\prime}(k)=\frac{2\left(1-k^{2}\right)}{k\left(1+k^{2}\right)^{2}} E(k)\left(\left(1-k^{2}\right) K(k)-E(k)\right) \text {. }
$$

It is well-known that $K(k)$ is an increasing function. Equality (22) implies that

$$
k\left(1-k^{2}\right) \frac{d K(k)}{d k}=E(k)-\left(1-k^{2}\right) K(k)>0 .
$$

Hence, the last factor in formula (24) is negative. Therefore, the right hand side of formula (24) is negative.

Corollary 4. The following inequality holds:

$$
2<I_{2}(a)<\frac{\pi}{\sqrt{2}}
$$

Proof. It is well-known that $E(k)<K(k)$. Therefore, according to formula (23), the function $I_{2}(b)$ is decreasing. Thus, one obtains the inequality

$$
2=I_{2}\left(\frac{\pi}{2}\right)<I_{2}(b)<I_{2}(0)=\frac{1}{\sqrt{2}} \int_{-1}^{1} \frac{d x}{\sqrt{1-x^{2}}}=\frac{\pi}{\sqrt{2}} .
$$

Proposition 14. For $b \in\left(0, \frac{\pi}{2}\right)$ the following inequality holds:

$$
\pi^{2} \frac{I_{1}(b)}{I_{2}(b)^{3}}<2
$$

Proof. The statement follows from Proposition 13 and the equality

$$
\pi^{2} \frac{I_{1}(0)}{I_{2}^{3}(0)}=2 .
$$

This completes the proof of the inequality $\lambda_{2 q-1}(0)<2$. 
3.5. Proof of the theorem. It follows from Theorem 1 that in order to prove Theorem 3 it is sufficient to prove that

$$
N(2)=2 q+4 p-2 \text { if } q \text { is odd }
$$

and

$$
N(2)=q+2 p-2 \quad \text { if } q \text { is even. }
$$

According to Proposition $10, \lambda_{0}(2)>2$. By Corollary 3 , one has $\lambda_{0}(l)>2$ as $l \geqslant 2$. Then due to Proposition $8, \lambda_{i}(l)>2$, when $l \geqslant 2$ and $k \geqslant 0$. For $l=0$, we get $\lambda_{2 q-1}(0)<\lambda_{2 q}(0)=2$. Hence, we have $2 q$ eigenfunctions of problem (18) with $l=0$ with eigenvalues less than 2. If $q$ is even, then we need to take into account the invariance under the transformation

$$
(\alpha, t) \longmapsto\left(\alpha+\pi, t+\frac{t_{0}}{2}\right) .
$$

The application of Proposition 12 for $n=2$ leaves $q$ eigenfunctions. By Proposition 8 one has $\lambda_{2 k+1}>\lambda_{2 k}$. Hence, for $l=1$ the following inequality holds, $\lambda_{2 p-2}(1)<$ $\lambda_{2 p-1}(1)=\lambda_{2 p-2}=2$. In the same way one obtains $2 p-1$ eigenfunctions if $q$ is odd and $p-1$ eigenfunctions if $q$ is even (here one should apply Proposition 11). According to Corollary 1, any eigenfunction of problem (18) with $l \geqslant 1$ provides exactly two eigenfunctions of the Laplace-Beltrami operator on $\widetilde{O}_{\frac{p}{q}}$. Thus, if $q$ is odd then one has $N(2)=2 q+2(2 p-1)=2 q+4 p-2$. If $q$ is even then one has $N(2)=q+2(p-1)=q+2 p-2$.

\subsection{The value of the corresponding functional.}

Proposition 15. If $q$ is odd then

$$
16 q \pi<\Lambda_{2 q+4 p-2}\left(\tilde{O}_{\frac{p}{q}}\right)=8 q \pi I_{2}(a)<4 \sqrt{2} q \pi^{2} .
$$

If $q$ is even then

$$
8 q \pi<\Lambda_{q+2 p-2}\left(\widetilde{O}_{\frac{p}{q}}\right)=4 q \pi I_{2}(a)<2 \sqrt{2} q \pi^{2} .
$$

Proof. By formula (17) for the metric $h$ on the torus $\tilde{O}_{\frac{p}{q}}$ one has $\sqrt{\operatorname{det} h}=\frac{1}{2 \pi}$. Hence the corresponding value of the functional is equal to

$$
2 \int_{0}^{t_{0}} \int_{0}^{2 \pi} \frac{1}{2 \pi} d \varphi d \alpha=2 t_{0} .
$$

For even $q$ one need to take into account the invariance under the transformation

$$
(\alpha, t) \longmapsto\left(\alpha+\pi, t+\frac{t_{0}}{2}\right),
$$


so this value has to be divided by two. Arguing as in Section 3.4 one obtains that

$$
t_{0}=2 q \int_{0}^{\frac{t_{0}}{2 q}} d t=4 q \pi I(b) .
$$

The application of Corollary 3.4 yields the desired inequalities.

3.7. Proof of Proposition 5. Let us consider $\Xi(b)$ as a function of $b \in\left(0, \frac{\pi}{2}\right)$. One has to prove that $\Xi(b)$ is decreasing on this interval. Let us begin with expressing $\Xi(b)$ in terms of elliptic integrals:

$$
\begin{aligned}
& \cos ^{2} b \int_{-b}^{b} \frac{1}{\cos \varphi \sqrt{\cos ^{4} \varphi-\cos ^{4} b}} d \varphi \\
& =\cos ^{2} b \int_{-\sin b}^{\sin b} \frac{1}{\left(1-y^{2}\right) \sqrt{\left(1-x^{2}\right)^{2}-\cos ^{4} b}} d \varphi \\
& =\cos ^{2} b \int_{-1}^{1} \frac{1}{\left(1-x^{2} \sin ^{2} b\right) \sqrt{1-x^{2}} \sqrt{1+\cos ^{2} b-x^{2} \sin ^{2} b}} \\
& =2 \frac{\cos ^{2} b}{\sqrt{1+\cos ^{2} b}} \Pi\left(\sin ^{2} b, \frac{\sin b}{\sqrt{1+\cos ^{2} b}}\right) \\
& =2 \frac{1-n}{\sqrt{2-n} \Pi\left(n, \sqrt{\frac{n}{2-n}}\right),}
\end{aligned}
$$

where $n=\sin ^{2} b$. Here the following changes of variables were used, $\sin \varphi=y$, $y=x \sin b$. Now the equality $\lim _{b \rightarrow 0} \Xi(b)=\frac{\sqrt{2}}{2} \pi$ follows from substituting $n=0$ into this formula. Using the formulae for the derivatives of $\Pi(n, k)$,

$$
\frac{\partial \Pi(n, k)}{\partial n}=\frac{1}{2\left(k^{2}-n\right)(n-1)}\left(E(k)+\frac{1}{n}\left(k^{2}-n\right) K(k)+\frac{1}{n}\left(n^{2}-k^{2}\right) \Pi(n, k)\right),
$$

and

$$
\frac{\partial \Pi(n, k)}{\partial k}=\frac{k}{n-k^{2}}\left(\frac{E(k)}{k^{2}-1}+\Pi(n, k)\right)
$$

one obtains

$$
\frac{d \Xi(n)}{d n}=\frac{1}{2 n \sqrt{2-n}}\left(E\left(\sqrt{\frac{n}{2-n}}\right)-K\left(\sqrt{\frac{n}{2-n}}\right)\right)<0 .
$$




\section{References}

[1] S. Bando and H. Urakawa, Generic properties of the eigenvalue of the Laplacian for compact Riemannian manifolds. Tôhoku Math. J. 35 (1983), 155-172. MR 0699924 Zbl 0534.58038

[2] M. Berger, Sur les premières valeurs propres des variétés riemanniennes. Compositio Math. 26 (1973), 129-149. MR 0316913 Zbl 0257.53048

[3] P. Byrd and M. Friedman. Handbook of elliptic integrals for engineers and scientists. Second ed., revised. Die Grundlehren der mathematischen Wissenschaften, Band 67. Springer Verlag, New York etc., 1971. MR 0277773 Zbl 0213.16602

[4] E. A. Coddington and N. Levinson, Theory of ordinary differential equations. McGrawHill, New York etc., 1955. MR 0069338 Zbl 0064.33002

[5] B. Colbois and J. Dodziuk, Riemannian metrics with large $\lambda_{1}$. Proc. Amer. Math. Soc. 122 (1994), 905-906. MR 1213857 Zbl 0820.58056

[6] A. El Soufi, H. Giacomini, and M. Jazar, A unique extremal metric for the least eigenvalue of the Laplacian on the Klein bottle. Duke Math. J. 135 (2006), 181-202. MR 2259925 Zbl 1109.58029

[7] A. El Soufi and S. Ilias, Immersions minimales, premiére valeur propre du laplacien et volume conforme. Math. Anal. 275 (1986), 257-267. MR 0854009 Zbl 0675.53045

[8] A. El Soufi and S. Ilias, Laplacian eigenvalue functionals and metric deformations on compact manifolds. J. Geom. Phys. 58 (2008), 89-104. MR 2378458 Zbl 1137.49040

[9] A. El Soufi and S. Ilias, Riemannian manifolds admitting isometric immersions by their first eigenfunctions. Pacific. J. Math. 195 (2000), 91-99. MR 1781616 Zbl 1030.53043

[10] A. Henrot, Extremum problems for eigenvalues of elliptic operators. Frontiers in Mathematics. Birkhäuser Verlag, Basel etc., 2006. MR 2251558 Zbl 1109.35081

[11] W.-Y. Hsiang and H. B. Lawson, Minimal submanifolds of low cohomogeneity. J. Diff. Geom. 5 (1971), 1-38. MR 0298593 Zbl 0219.53045

[12] D. Jakobson, N. Nadirashvili, and I. Polterovich, Extremal metric for the first eigenvalue on a Klein Bottle. Canad. J. Math. 58 (2006), 381-400. MR 2209284 Zbl 1104.58008

[13] K. Kenmotsu, A characterization of bipolar minimal surfaces in $\$^{4}$. Tôhoku Math. J. (2) 26 (1974), 587-598. MR 0361994 Zbl 0332.53033

[14] S. Kobayashi and K. Nomizu, Foundations of differential geometry. Vol. II. Interscience Publishers, John Wiley \& Sons, New York etc., 1969. MR 0152974 Zbl 0175.48504

[15] N. Korevaar, Upper bounds for eigenvalues of conformal metrics. J. Differential Geom. 37 (1993), 73-93. MR 1198600 Zbl 0794.58045

[16] H. Lapointe, Spectral properties of bipolar minimal surfaces in $\$^{4}$. Differential Geom. Appl. 26 (2008), 9-22. MR 2393969 Zbl 1139.58025

[17] H. B. Lawson, Complete minimal surfaces in $\$^{3}$. Ann. of Math. (2) 92 (1970), 335-374. MR 0270280 Zbl 0205.52001

[18] N. Nadirashvili, Berger's isoperimetric problem and minimal immersions of surfaces. Geom. Funct. Anal 6 (1996), 877-897. MR 1415764 Zbl 0868.58079 
[19] T. Otsuki, Minimal hypersurfaces in a Riemannian manifold of constant curvature. Amer. J. Math. 92 (1970), 145-173. MR 0264565 Zbl 0196.25102

[20] A. V. Penskoi, Extremal spectral properties of Otsuki tori. Math. Nachr. 286 (2013), 379-391. MR 3028782 Zbl 1271.58007

[21] A. V. Penskoi, Extremal spectral properties of Lawson tau-surfaces and the Lamé equation. Moscow Math. J. 12 (2012), 173-192. MR 2952430 Zbl 1272.58010

[22] P. C. Yang and S.-T. Yau, Eigenvalues of the laplacian of compact Riemann surfaces and minimal submanifolds. Ann. Scuola Norm. Sup. Pisa Cl. Sci. 7 (1980), 55-63. MR 0577325 Zbl 0446.58017

Received May 17, 2012

Mikhail A. Karpukhin, Department of Geometry and Topology,

Faculty of Mechanics and Mathematics, Moscow State University,

Leninskie Gory, GSP-1, 119991, Moscow, Russia

Independent University of Moscow, Bolshoy Vlasyevskiy pereulok 11, 119002, Moscow, Russia

E-mail: karpukhin@mccme.ru 